International Journal of Network Security \& Its Applications (IJNSA), Vol.3, No.4, July 2011

\title{
A Novel SCHEME For DeVIATION DETECTION IN ASYNCHRONOUS Distributed PRICING
}

\author{
S.S. Prasad ${ }^{1}$, Rithika Baruah ${ }^{2}$ and Siddharth. ${ }^{3}$ \\ Department of Electronics \& Communication Engineering, National Institute of \\ Technology, Jamshedpur, India \\ ${ }^{1}$ ssprasadeieee.org \\ ${ }^{2}$ rithika_baruah@ieee.org \\ ${ }^{3}$ sidaudhi@gmail.com
}

\begin{abstract}
Modelling resource allocation problems in the form of non-cooperative pricing games takes into account the difference between how much a given performance metric is valued and how much is paid for it. For the convergence of the sum of all users' payoff to a global maximum, the determination of the utility function is essential. Although supermodularity conditions have been previously defined and determined to obtain suitable utility functions, different utilities have significantly varying performance characteristics under similar network parameters. In an ad-hoc framework, absence of a central authority leads to uncontrollability of unfairness. Users could misbehave by broadcasting high price coefficients to force other users to transmit at a lower power. This paper proposes an adaptation of the Asynchronous Distributed Pricing Algorithm with a Deviation Detection Block that re-aligns the deviated system back into the algorithm.
\end{abstract}

\section{KEYWORDS}

Game theory, asynchronous distributed pricing, distributed resource allocation, deviation detection.

\section{INTRODUCTION}

Wireless ad hoc networks are 'impromptu' networks comprising of autonomous users with individually distinguishable Quality of Service (QoS) requirements. Applications of ad hoc networks include ubiquitous commercial usage as well as emergency applications. A distributed process is one that is not carried out centrally, but independently at every node. When resources such as power, bandwidth, etc are allocated to a system of independent nodes having no central unit controlling them (ad-hoc networks), the process is called Distributed Resource Allocation. In order to arrive at a distributed process for resource allocation, challenges such as conflict and non-cooperation among nodes must be overcome. Normally, nodes in this network do not function on the principles of altruism and pursue individual goals. In the absence of a central controller or algorithm with incentive mechanisms, performance degradation occurs in such networks. One such scheme ensuring throughput fairness in the entire network is the Asynchronous Distributed Pricing (ADP) suggested by Schmidt et al. in [1] rooted in the principles of game theory.

Game theory, a branch of applied mathematics, deals primarily with decision making situations with properly defined utilities. Game theory models individual, independent decision makers whose actions potentially affect all other decisions in the game. Conflict and cooperation between intelligent rational decision makers as defined in [2] is primarily replicated in game theoretical calculations. Such situations are modeled on players which are assumed to be rational and selfish, the precise definitions of which will be elaborated in the sections that follow. The 
analytical tools in this theory are based on the assumption of strict adherence to a strategy based on perceived goals. Game theory can be used to model existing systems as a particular type of game in order to find optimum solutions to the models or better the performance of the models. These models can also be used to study the properties of the system. Moreover, game theory can also be used to implement new models to improve the system because in this case the objectives of the game the fixed and the game is designed so as to attain the predefined goal set.

Primarily, game theory was invented with applications in economics although today, it finds many applications including wireless engineering. Nowadays, game theoretic analysis has been used in ad hoc networks in a wide range of applications. Examples include algorithms for power control and waveform adaptation in the physical layer, medium access control as well as routing in the network layer besides others [3, 4]. The pricing algorithms have been used for resource allocation games ranging from bandwidth allocation, distributed beamforming, interference pricing algorithms to power control games using virtual currency in [5-8]. This paper deals with the problem of fair play among network entities chosen for a power control game aiming at efficient resource allocation in ad hoc networks. Figure 1 depicts the evolutionary process of game theory in wireless ad hoc networks.

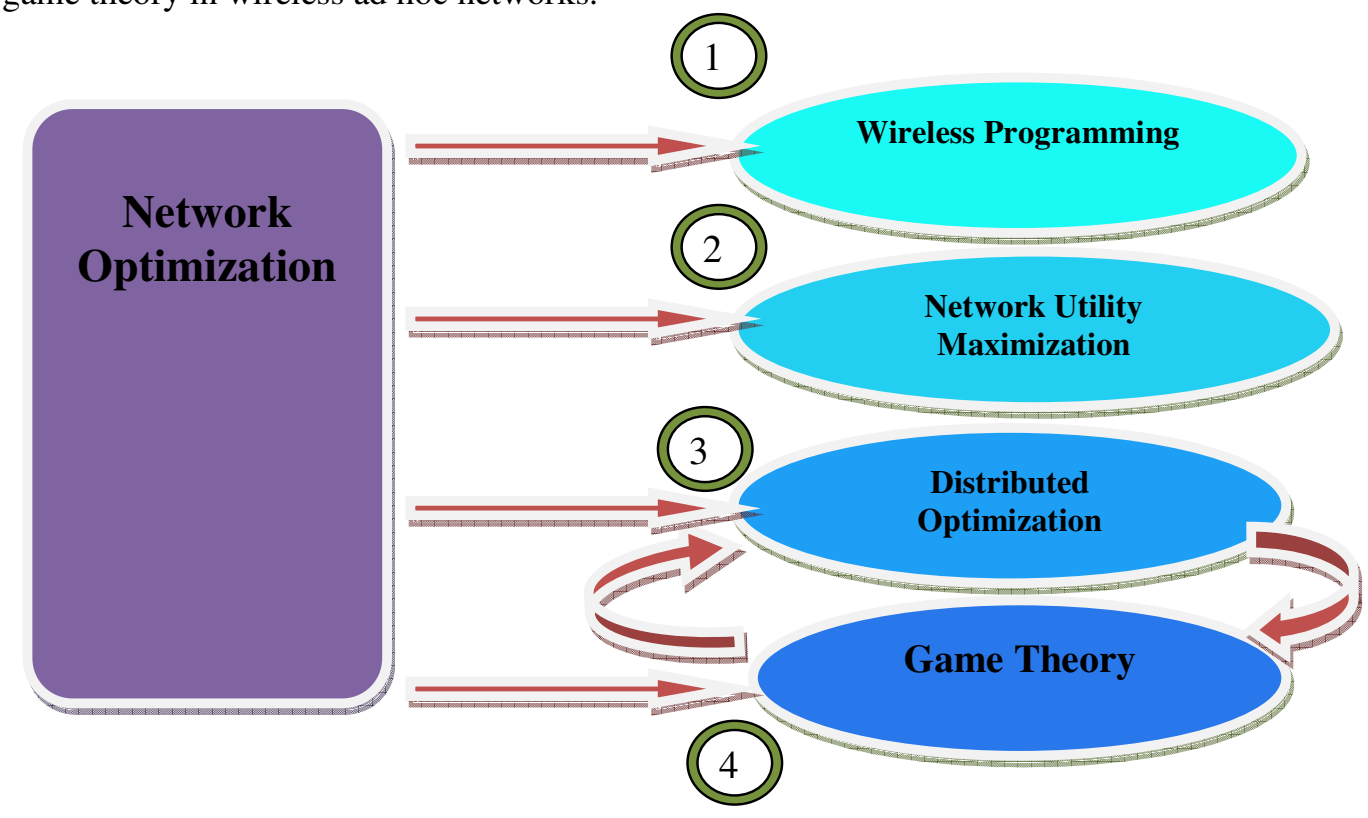

Figure 1. Evolution of game theoretical applications for network optimization

The more the networks evolve, the more they tend to move towards decentralization without a base station controlling the amount of power or the band of frequencies for operation of the nodes of the network. E.g. wireless sensor networks, mobile ad hoc networks, and pervasive computing. These networks are self-organizing multihop networks. Thus distributed decision making shall potentially become the most important field of research in communications taking network conditions as well as channel conditions into account as used in our thesis.

In C. H. Papadimitriou [16], the operation called price of anarchy was introduced which is the difference of performance between selfish, local goal oriented networks and communal mode of operation. Game theory can be used to assess this cost. In this thesis, we have used the case of perfect information where all players know each others' utilities and there is a certain goal that each node tries to acquire, the goal of maximum utility. Future directions in this research include the area of the type of games called, games of imperfect information which would rather focus on 
Bayesian equilibrium than Nash equilibrium. Cheating can be detected with the help of this model of ignorance about other nodes as used in L. A. DaSilva and V. Srivastava [17].

Another model accounting for uncertainties regarding other players' strategies are called games of imperfect monitoring. Each player observing the actions of every other player at the end of each stage in a repeated game is challenged in this type of games. E.g. in ad hoc networks, nodes can deny forwarding packets for others to conserve their limited resources and monitoring also requires and thus, forwarding of packets is not necessarily feasible. Instead of monitoring other players' actions, the nodes observe a random public signal at the end of every stage of the game which is correlated to the actions of all the other players in the game. Distribution function of the public signal depends on the action profile chosen by the other players but does not deterministically reveal other players' actions. In future, these games can be elaborately researched upon. Another research area which is still in its infancy is the area of privately monitored games where each player has a distinct assessment of others' actions in a repeated game format.

Asynchronous Distributed Pricing scheme addresses this problem by designing autonomous allocation methods in each transmitter, based on the exchange of virtual interference prices. User demands for service and associated priorities depend on a certain QoS metric as stated earlier as well. It is known as the utility of the network. The network objective is then the sum utility over all users. This objective is flexible enough to accommodate a wide range of performance metrics through an appropriate assignment of utility functions. Each interference price is associated with a particular receiver and indicates the marginal decrease in utility due to a marginal increase in interference. Given a set of interference prices from nearby receivers, a transmitter then selects resources according to a best response which maximizes its utility minus the total interference cost. Applications of game theory to networking typically assume non-cooperative users, and thus, these scenarios fit naturally within game theoretic frameworks. The motivation in ADP to use game theory is to model the preferences of each user so that best response updates in the resulting game lead to convergence to an optimal (utility-maximizing) allocation. The network objective of finding a global optimum solution for the maximum sum utility over all users accommodates a wide range of QoS metrics. At Nash equilibrium of a game, both the power and price player chose not to deviate. The convergence of ADP algorithm, thus, can be ascertained by showing that the best response updates of the game converge.

Our paper considers efficient resource allocation in ad hoc networks and hence, we deal with distributed algorithms. Shi et al. in [8] have shown that ADP converges within the least possible number of iterations as compared to previous distributed algorithms. However, for the class of supermodular games defined and explained in [9], best response updates converge even when the algorithm for power or price update is arbitrarily asynchronous. ADP has been proved to contain a global optimum for supermodular games in [10].

In a game, selfish nodes try to maximize their own utilities by not considering the benefaction of other nodes in the network. However, another group of nodes in a network exists in which the nodes are called malicious nodes. These nodes try to harm the operation of the network by not playing the game according to its rules. In a centrally monitored game, unfairness can be easily avoided. However, selfishness modeled in a non-cooperative game theoretic with a distributed framework can lead to uncontrollability of unfairness without extrinsic incentive mechanisms. Virtual pricing is one such mechanism. In order to ensure cooperation in ad hoc networks, the concept of nuglets was first introduced in [11]. Subsequently, they found applications in the Terminodes project [12]. The 'confidant' protocol detected misbehavior by tracking of the neighborhood nodes by each node and reporting misbehaviors to central controller as explained in [13]. Validity of these reports is evaluated according to trust records in the reputation manager. The 'core' protocol designed by Michiardi and Molva in [14] also functioned in similar lines. In 
this protocol reputation values were assigned to other nodes at each node in their own separate reputation tables which were maintained by the nodes according to their own observations. Thus, misbehavior would lead to isolation and non-cooperation from other nodes in the game.

A set of coefficients of virtual price for the network which is an entity that can be regarded as a payment for a desired payoff which varies with the interference that a terminal creates in the network is mostly used in payment-based schemes. Users generating higher interference pay more by transmitting at a lower power to give other users a fair throughput share. Some players may, however, misbehave by broadcasting higher price coefficients so as to force other players to transmit at a lower power. One type of games classified as the Prisoner's Dilemma game, can be used to provide incentives to the players to play fairly in these distributed games [15]. This study has considered repeated games and fair broadcasting of the prices is ensured by incorporation of a particular methodology that this study has introduced in the ADP algorithm. This paper also aims to prove this analytically and hence improve the payoff of the players in a wireless game by avoiding cheating even in a distributed framework.

\section{Problem Formulation}

There are two entities in the ADP algorithm that are used for optimization - transmission power $P$ and interference price $\pi$. Each receiver announces a single interference price $\left(\pi_{i}\right)$, which is the marginal cost of his own utility $\left(u_{i}\right)$ per unit interference. This is mathematically given as:

$$
\pi_{i}=-\frac{d}{d I_{i}}\left(u_{i}\right)
$$

Where $I_{i}$ is the total interference power at $i^{\text {th }}$ receiver.

While the price $\left(\pi_{i}\right)$ acts as a factor of the negative externalities, the new payoff function can be determined by:

$$
\prod\left(P_{i}, \pi_{j}\right)=u_{i}-\sum_{j \neq i} \pi_{j} h_{j i}^{2} P_{i}
$$

By maximizing the resultant payoff $\prod\left(P_{i}, \pi_{j}\right)$ each node maximizes its own utility and minimizes the interference caused to other nodes in the system.

For the ADP algorithm, if the coefficients of relative risk aversion of each user's utility satisfy

$$
1<=C R_{k}(\operatorname{SINR})<=2
$$

for all feasible SINRs, then the resulting game is supermodular. Moreover, this case has a unique global optimum and so it follows that the ADP algorithm will globally converge to the optimum power allocation.

Hence, chosen utility functions must be supermodular to guarantee convergence of the algorithm. One such example of a utility function that makes the resulting game supermodular is $-1 / x$, with $\mathrm{CR}_{\mathrm{k}}(\mathrm{x})$ value of 2 .

The complete ADP algorithm is shown below in Figure 2. 


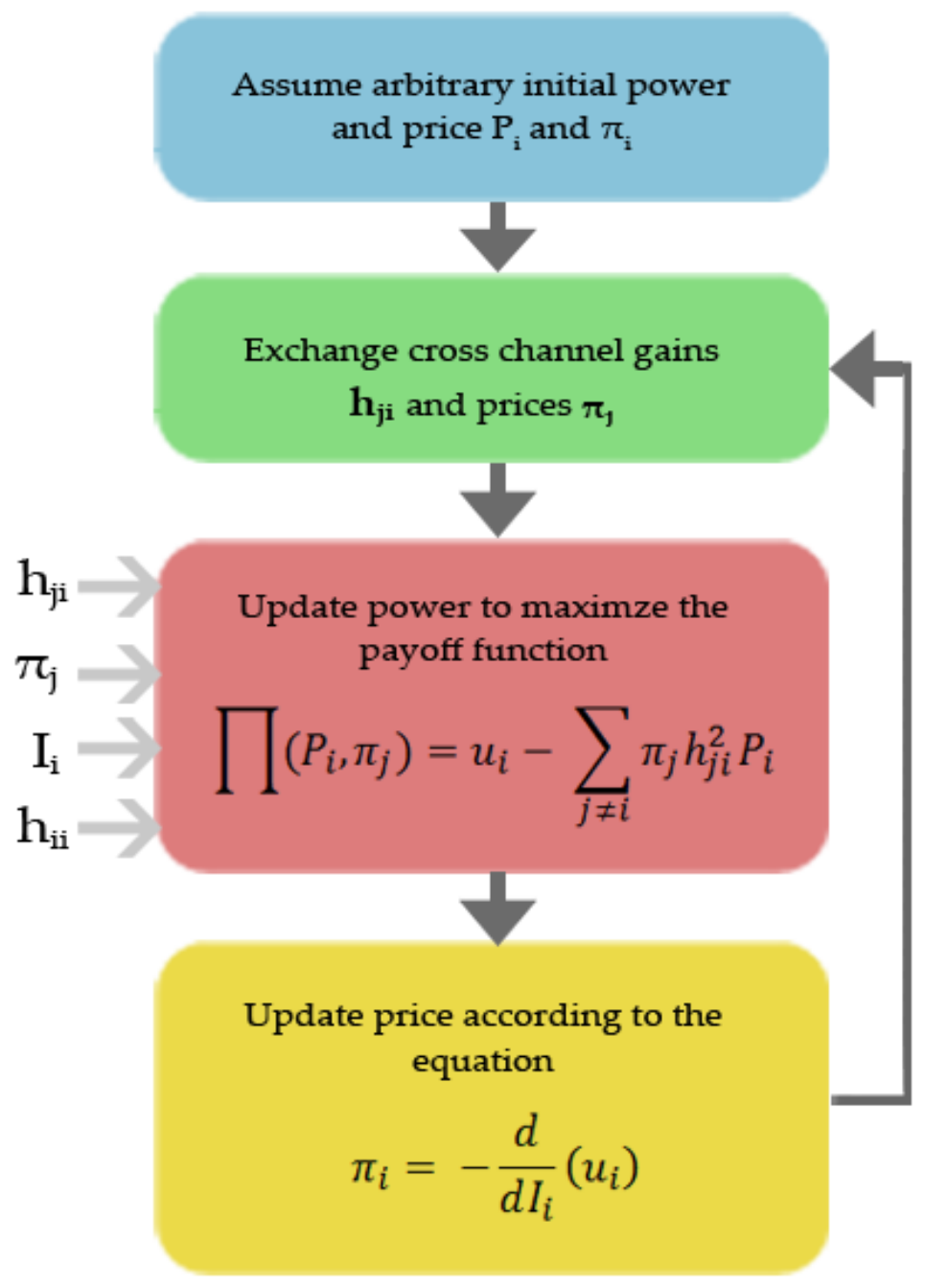

Figure 2. Flowchart of the ADP algorithm

In the ADP algorithm, the users iteratively adapt their power allocations and announce new interference prices. When a user adapts its power, it maximizes the resulting payoff assuming that the power allocations and interference prices of the other users are fixed. The power update is therefore the best response of an agent in the resulting game.

ADP being a distributed algorithm, each node in the network acts autonomously and updates its utility asynchronously according to the network statistics. The network objective in ADP is basically finding a global optimum solution for the maximum sum utility over all users, accommodating a wide range of QoS metrics.

The information exchange (overhead) required for the algorithm is very limited. For every node, the transmitter needs to know the interference prices from other receivers, its own SINR, and the cross-channel gains to neighboring receivers. It does not need to know the other channel gains in the network and other users' utility functions. Also, to compute the interference price, the receiver must know the direct channel gain and the interference-plus-noise power (or equivalently, the SINR and the transmitted power). 
Asynchronous Distributed Pricing is a distributed algorithm and hence, each node acts autonomously and each node updates its utility asynchronously according to the network statistics. The network objective in this problem is to find a global optimum solution for the maximum sum utility over all users.

Every receiver declares its own interference price in the network which indicates the marginal decrease in utility due to a marginal increase in interference. A transmitter selects power according to a best response, which maximizes its utility minus the cost of interference incurred. Users iterate between price and power updates until the algorithm has converged. The selfish nature of nodes leads to false reporting of prices, resulting in unfair resource distribution wherein nodes broadcast higher price coefficients so as to force other players to transmit at a lower power.

\section{System Framework and Mathematical Perspective}

A game is a set consisting of the following entities: a finite number of players or decision makers $(\mathrm{N})$, a set of possible actions of the players $\left(\mathrm{A}_{\mathrm{i}}\right)$, and the set of utilities or consequences of the actions $\left(\mathrm{U}_{\mathrm{i}}\right)$.

$$
G=\left\langle N,\left\{A_{j}\right\}_{j \in N},\left\{U_{j}\right\}_{j \in N}\right\rangle
$$

The decision makers ensure the best possible utility calculated according to their preferences while most of the games are designed so that the sum utility of all the players is a global maximum. The preferences of the players are usually shown with the help of utility functions.

One of the most important solution concepts of a game which the game usually converges to is the Nash equilibrium. Nash equilibrium is the set of actions from which no player deviates or tends to deviate unilaterally because none of the players can benefit by changing strategies when other players' strategies are the same. But Nash equilibrium may or may not be the best cumulative payoff. A system can be designed so as to converge the set of strategies to the best cumulative payoff as well as the Nash equilibrium in a non-cooperative framework so that the solution set reached to by the game is the best possible one as used in the game we have used in this thesis.

An action vector $a^{*}=a_{1} *, a_{2} *, a_{3} *, \ldots \ldots \ldots a_{n} *$ is a Nash equilibrium if

$$
\mathrm{u}_{\mathrm{i}}\left(\mathrm{a}_{\mathrm{i}}^{*}, \mathrm{a}_{-\mathrm{i}}^{*}\right) \geq \mathrm{u}_{\mathrm{i}}\left(\mathrm{a}_{\mathrm{i}}, \mathrm{a}_{-\mathrm{i}}^{*}\right) \text { for all } \mathrm{a}_{\mathrm{i}} \text { and } \mathrm{u}_{\mathrm{i}}
$$

We consider the following network framework for the problem. The network consists of $\mathrm{N}$ "users" where each "user" is a single transmitter/receiver pair and each transmitter uses same bandwidth. Each receiver is interested in the signal from its associated transmitter only. The disturbance to the message signals comes from all other transmitters which constitute the interference. In addition to interference, all the receivers experience equal amount of background noise. The conditions of the wireless channel are reflected in the channel gains between each transmitter and each receiver.

We have considered a system of 3 transmitter-receiver pairs with channel matrices $h_{i j}$ where $i$ is the receiver and $\mathrm{j}$ is the transmitter. The system model is shown in Figure 3. 


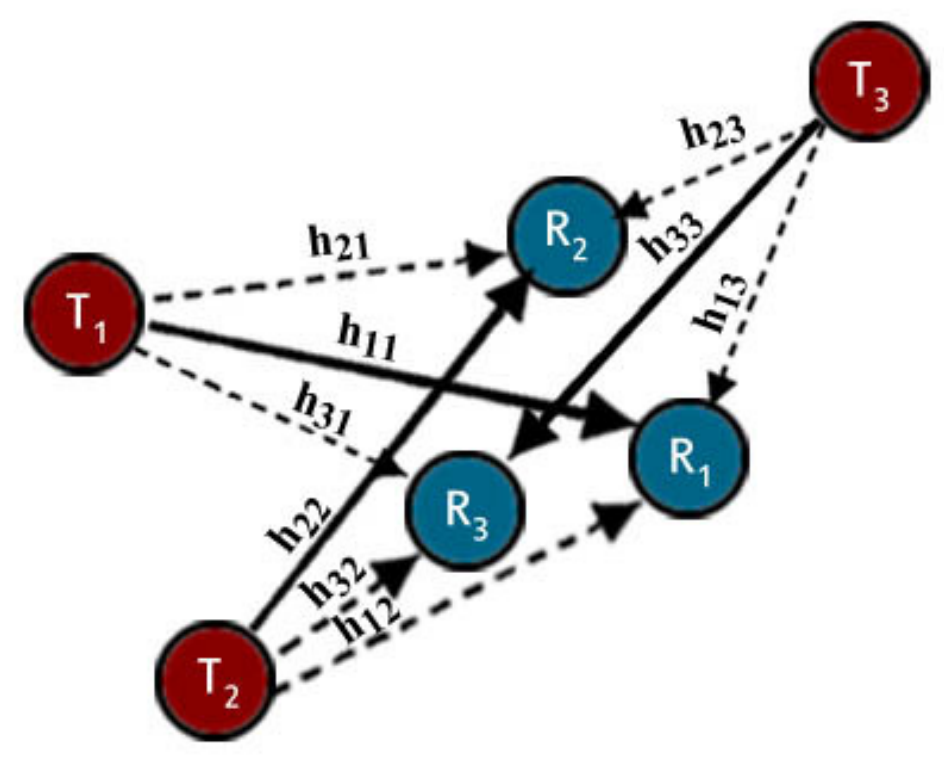

. Figure 3. System model and channel gains

We assume perfect channel estimation for all the nodes in our model. The channel is modeled as a lossy channel for SINR calculations and SIR calculations. Each user needs to know adjacent channel gains and interference prices only. This decreases the overall information exchange overhead. User's QoS are preferences given by a utility function $u_{i}\left(R_{i}(P)\right)$ where $u_{i}(\cdot)$ is increasing, twice differentiable and sufficiently concave function of $\mathrm{R}_{\mathrm{i}}$. Sufficient concavity is defined in [18] which is modeled in supermodular games relies on the constraint that the utility must neither be non-concave nor too concave.

Common QoS metrics used to perceive a user's performance in a network are the received SIR and SINR. ADP algorithm utilizes a virtual currency scheme to localize the optimization problem faced in maximizing the sum utility of the network, by allowing the nodes to autonomously solve the power optimization problem constrained by the strategy of the game and maximum of the principal entity which is the transmitting power in a power game.

The resulting payoff function for power optimization suggested by the algorithm is,

$$
\Pi\left(P_{i}, \pi_{j}\right)=u_{i}-\sum_{j \neq i} \pi_{j} h_{j i}^{2} P_{i}
$$

where $\prod$ is the net payoff, $u_{i}$ is the utility function, $\prod_{j}$ is the price announced by the $j^{\text {th }}$ receiver, $h_{j i}$ is the cross channel gain between the $i^{\text {th }}$ transmitter and the $j^{\text {th }}$ receiver and $P_{i}$ is the power transmitted by the $i^{\text {th }}$ transmitter.

The interference price is a virtual quantity which is the marginal cost of a user's own utility per unit interference as is given by:

$$
\pi_{i}=-\frac{d}{d I_{i}}\left(u_{i}\right)
$$


International Journal of Network Security \& Its Applications (IJNSA), Vol.3, No.4, July 2011

The choice of utility function is essential for the convergence of the algorithm. In [19], J. Yuan and $\mathrm{W}$. Yu have shown that the ADP power game becomes supermodular if the coefficient of relative risk aversion factor lies between 1 and 2, resulting in a global optimum solution for the sum utility.

We chose two main utility functions, which satisfy the necessary condition of supermodularity, $\log (\mathrm{x})$ with $\mathrm{CR}_{\mathrm{k}}(\mathrm{x})=1$ and $-1 / \mathrm{x}$ with $\mathrm{CR}_{\mathrm{k}}(\mathrm{x})=2$. However, in [20] it has been shown that rate utilities also converge subject to a few constraints on the ADP algorithm. We consider a diagonally dominant channel and the convergence thus obtained is a local optimum which may be multiple depending upon the test cases considered. Also, we compared the results of the utilities for both SIR and SINR service metrics.

\subsection{Case $I: u(x)=\log (x)$}

As the optimization problem is solved locally, for individual nodes, the variable parameter is $P_{i}$

$$
\begin{gathered}
\prod\left(P_{i}, \pi_{j}\right)=\log \left(a_{i} P_{i}\right)-\sum_{j \neq i} \pi_{j} h_{j i}^{2} P_{i} \\
\prod\left(P_{i}, \pi_{j}\right)=\log \left(a_{i} P_{i}\right)-b_{i} P_{i} \\
\text { where } a_{i}=\frac{h i i^{2}}{\sum_{j \neq i} h i j^{2} P j+\sigma^{2}} \text { and } b_{i}=\sum_{j \neq i} \pi_{j} h_{j i}^{2}
\end{gathered}
$$

Maximizing (3), the condition for power update in ADP algorithm is,

$$
P_{i}=\frac{1}{b_{\mathrm{i}}}
$$

It is notable that the interference price broadcasted by the user is equal to the interference power seen by it. In case of SINR based utility functions, the price is given by the inverse of the sum of the interference power and the noise variance at the receiver $\left(\sigma^{2}\right)$.

$$
\boldsymbol{\pi}_{\boldsymbol{i}}= \begin{cases}\frac{1}{\boldsymbol{I}_{\mathbf{1}}} & \text { for SIR based } \\ \frac{\mathbf{1}}{\boldsymbol{I}_{\mathbf{1}}+\boldsymbol{\sigma}^{\mathbf{2}}} & \text { for SINR based }\end{cases}
$$

\subsection{Case I: $u(x)=-1 / x$}

Re-writing (1) in terms of the local variable $P_{i}$

$$
\prod\left(P_{i}, \pi_{j}\right)=-\frac{1}{a_{i} P_{i}}-b_{i} P_{i}
$$


International Journal of Network Security \& Its Applications (IJNSA), Vol.3, No.4, July 2011

$$
\text { where } a_{i}=\frac{h i i^{2}}{\sum_{j \neq i} h i j^{2} P j+\sigma^{2}} \text { and } b_{i}=\sum_{j \neq i} \pi_{j} h_{j i}^{2}
$$

Maximizing (4),

$$
=>P_{i}=\frac{1}{\sqrt{a_{i} b_{i}}}
$$

The parameter $a_{i}$ incorporates the noise variance factor in case of SINR based utility function. The interference price of the user is found to be independent of its interference power and noise, and is given by:

$$
\pi_{i}=\frac{1}{h_{i i}^{2} P_{i}}
$$

From Eqn.6. we have obtained an expression for the value of interference price of any receiver for the $-1 / x$ utility function. The successful computation of this price requires the computing node to know the transmission power and the direct channel gain of the $i^{\text {th }}$ node.

With the help of this expression, this study modelled a deviation detection block shown in Figure 4 , which can be executed by any node subject to certain conditions. This block is executed only when the change in the reported price of a node exceeds a specific threshold value, which is computed by comparing the change in price of other nodes. The block compares the value of the price computed internally, from Eqn. 6, and the value of the price reported by the node under investigation. Deviation can be ascertained when both the values do not match.

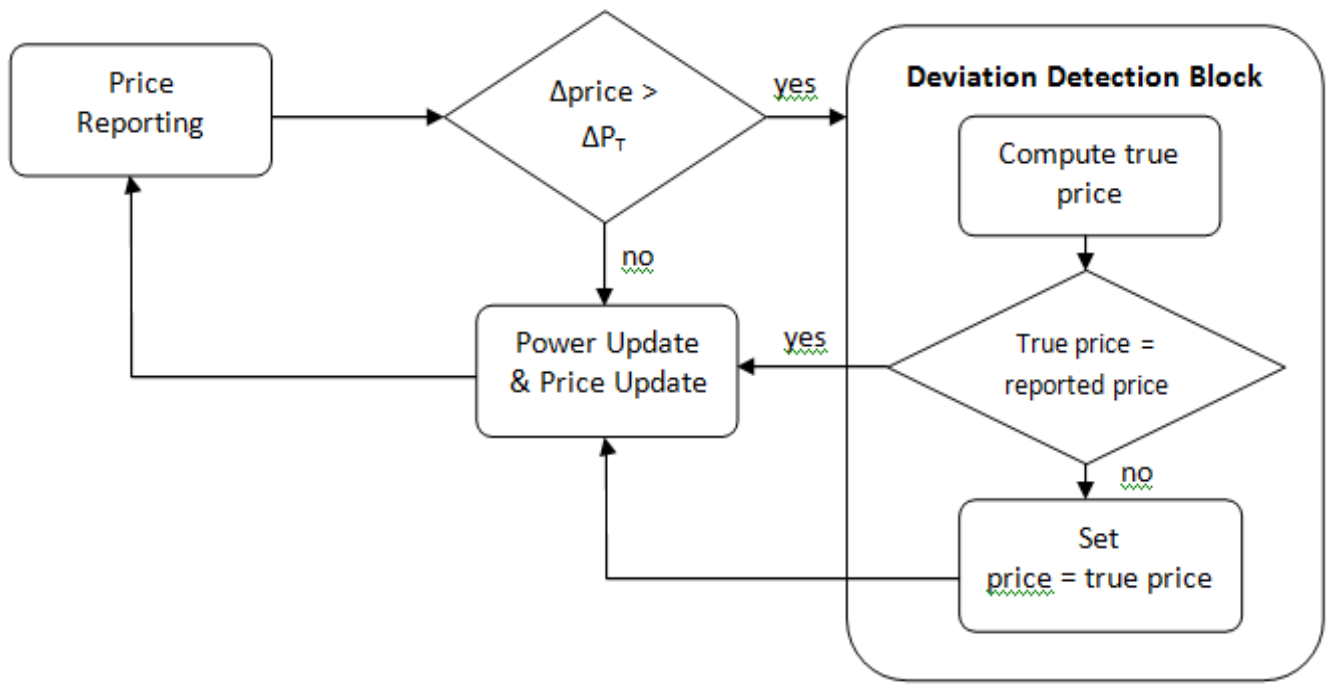

Figure 4. Flowchart for Deviation detection

Once cheating is detected, the algorithm is set back on track using the true value of interference price computed by the cheating detection block. The threshold value of price change can be set as the mean difference in all the other truthfully reported prices.

$$
\Delta p_{T}=\frac{\sum_{j=1, j \neq i}^{N} \Delta \pi_{j}}{N}
$$


International Journal of Network Security \& Its Applications (IJNSA), Vol.3, No.4, July 2011

where $p_{T}$ is the threshold value of interference price, $N$ is the number of truthfully reported prices and is the price reported by the $\mathrm{j}^{\text {th }}$ node and the deviating node is the $\mathrm{i}^{\text {th }}$ node.

This paper has assumed that the number of deviating nodes is very less compared to the total number of nodes in the system, as the threshold value of reported prices is more accurate when the $N$ is large. One important point to note is that the deviation detection block incorporated does not change the course of ADP algorithm; rather it simply re-aligns it back to its original path. Hence, the convergence of the adapted ADP algorithm still holds.

Achieving maximum performance out of such a system involves in controlling the nodes' selfishness where they deviate from the algorithm to achieve higher individual performance but degrading the performance of the system. The $-1 / x$ utility function presented an opportunity for detecting such deviations. The theoretical block proposed for cheating detection makes use of limited overhead and information exchange in order to detect any node's deviation from the algorithm and re-align the system to the actual ADP algorithm.

\section{Conclusions}

The game theoretical approach to QoS based distributed resource allocation acts as a preferable alternative to the centralized scheme owing to its advantages of reduced overhead and information exchange. Applications of these networks are found in ubiquitous civilian and commercial usage, where nodes typically belong to different authorities and may not pursue a common goal. Achieving maximum performance out of such a system involves in controlling the nodes' selfishness where they deviate from the algorithm to achieve higher individual performance but degrading the performance of the system. The use of $-1 / x$ utility function in asynchronous distributed pricing algorithm presented an opportunity for detecting such deviations. The theoretical block proposed for cheating detection makes use of limited overhead and information exchange in order to detect any node's deviation from the algorithm and re-align the system to the actual ADP algorithm. The limitations of this approach to fair power control in ad-hoc networks is that the information exchange involved in the deviation detection block is higher than that of the unchanged ADP algorithm. Also, its utility specific nature makes the use of $-1 / x$ utility function mandatory.

\section{REFERENCES}

[1] D. Schmidt, C. Shi, R.A. Berry, M. Honig, and W. Utschick, "Distributed Resource Allocation Schemes," in IEEE Signal Processing Magazine, vol. 26, no. 5, pp. 53-63, September 2009.

[2] Roger B. Myerson, "Game Theory: Analysis of Conflict”, Harvard University Press, 1991.

[3] J. Hicks, A. MacKenzie, J. Neel, J. Reed, "A game theory perspective on interference avoidance," in IEEE Global Telecommunications Conf., vol. 1, pp. 257-261, December 2004.

[4] V. Srivastava, J. Neel, A.B. Mackenzie, R. Menon, L.A. Dasilva, J.E. Hicks, J.H. Reed, and R.P. Gilles, "Using game theory to analyze wireless ad hoc networks," in IEEE Communications Surveys \& Tutorials, vol. 7, no. 4, pp. 46-56, 2005.

[5] Y. Qiu and P. Marbach, "Bandwidth allocation in ad hoc networks: A price-based approach," in Proceedings of IEEE INFOCOM, vol. 2, 2003, pp. 797-807.

[6] Y. Xue, B. Li, and K. Nahrstedt, "Price based resource allocation in wireless ad hoc networks," in Proceedings of 11th International Workshop on Quality of Service, 2003.

[7] C. Shj and M.L. Honig, "Local interference pricing for distributed beamforming in MIMO networks," in Proc. Military Communications Conference, 2005, pp. 1-9, Oct 2009. 
International Journal of Network Security \& Its Applications (IJNSA), Vol.3, No.4, July 2011

[8] C. Shi, D. A. Schmidt, R. A. Berry, M. L. Honig, and W. Utschick, "Distributed Interference Pricing for the MIMO Interference Channel," in Proc. IEEE International Conference on Communications, June 2009.

[9] V. V. Veeravalli, "Interference Management in Wireless Networks," in Qualcomm Seminar, San Diego, CA, February 2008.

[10] S. Agarwal, S. Krishnamurthy, R. Katz, and S. Dao, "Distributed power control in ad-hoc wireless networks," in International Symposium Personal, Indoor and Mobile Radio Communications, pp. F-59-F-66, 2001.

[11] L. Blazevic, L. Buttyan, S. Capkun, S. Giordiano, J.P. Hubaux, and J.Y. Le Boudec, "Self Organization in mobile ad-hoc networks: the approach of terminodes", in IEEE Communications Magazine, 39(6):166-174, June 2001

[12] Http://www.terminodes.org [20 $0^{\text {th }}$ October 2003]

[13] S. Buchegger and J. Le Boudec, "Performance analysis of the CONFIDANT protocol", in Proceedings of IEEE/ACMWorkshop on Mobile Ad Hoc Networking and Computing (MobiHOC), June 2002.

[14] P. Michiardi and R. Molva, "Core: A Collaborative reputation mechanism to enforce node cooperation in mobile ad hoc networks," in Communication and Multimedia Security Conference, 2002.

[15] C.K. Tan, M.L. Sim and T.C. Chuah, "Fair power control for wireless ad-hoc networks using game theory with pricing scheme," in IET Communications, 2010, Vol. 4, Issue No. 3, pp. 322-333.

[16] C. H. Papadimitriou, "Algorithms, games and the internet," in Proceedings of the $33^{\text {rd }}$ ACM Symposium on Theory of Computing, 2001, pp. 749-753.

[17] L. A. DaSilva and V. Srivastava, "Node participation in ad-hoc and peer-to-peer networks: A game-theoretic formulation," in Workshop on Games and Emergent Behavior in Distributed Computing Environments, Birmingham, U K, September 2004.

[18] J. Huang, R. A. Berry, and M. L. Honig, "Distributed interference compensation for wireless networks," in IEEE Journal on Selected Areas in Communications, vol. 24, no. 5, pp. 1074-1084, May 2006.

[19] H. Boche, S. Naik, and T. Alpcan, "Concave resource allocation problems for interference coupled wireless systems," in IEEE International Conference on Acoustics Speech and Signal Processing (ICASSP), 2010, pp. 3002-3005, March 2010.

[20] S. Stanczak, M. Wiczanowski and H. Boche, "Distributed Utility-Based Power Control: Objectives and Algorithms," in IEEE Transactions on Signal Processing, vol 55, no. 10, pp. 50585068, October 2007.

[21] J. Huang, R. A. Berry, and M. L. Honig, "Performance of Distributed Utility-Based Power Control for Wireless Ad Hoc Networks," in Proc. Military Communications Conference, 2005, pp. 24812487, Oct 2005.

[22] C. Shi, R.A. Berry, and M.L. Honig, "Distributed interference pricing for OFDM wireless networks with non-separable utilities," in Conference on Information Sciences and Systems, CISS 2008, pp. 755-760, March 2008.

[23] D. M. Topkis, "Supermodularity and Complementarity," Princeton Univ. Press, 1998.

[24] C. Shj and M.L. Honig, "Monotonic convergence of distributed interference pricing in wireless networks," in IEEE International Symposium on Information Theory 2009, pp. 1619-1623, JuneJuly 2009. 